\title{
PAPER
}

\section{Frequency domain method to estimate the coefficients of feedback control filter for active noise control systems}

\author{
Yusuke Iwamatsu ${ }^{1, *}$, Kensaku Fujii ${ }^{1, \dagger}$ and Mitsuji Muneyasu ${ }^{2, \ddagger}$ \\ ${ }^{1}$ Department of Computer Engineering, University of Hyogo \\ ${ }^{2}$ Faculty of Engineering, Kansai University
}

(Received 27 October 2005, Accepted for publication 7 April 2006)

\begin{abstract}
In this paper, we propose a method for repeatedly updating the coefficients of the feedback control filter used for canceling a feedback path from a loudspeaker to a noise detection microphone. The coefficients are usually estimated by feeding a sequence of extra noise to the loudspeaker. However, feeding such extra noise under active noise control is undesirable. The proposed method estimates the coefficients without feeding the extra noise. Concretely the proposed method provides two independent equations using the estimation errors involved in the coefficients of the noise control filter and then estimates the coefficients by solving those equations. One problem is that the feedback component is extremely small in comparison with the primary noise incident on the noise detection microphone. In this case, estimation of the coefficients is difficult. In this work. we solves this problem by estimating first the primary noise and then the feedback path.
\end{abstract}

Keywords: Active noise control, Feedback control filter, Simultaneous equations method, Frequency domain, Linear prediction

PACS number: $43.50 . \mathrm{Ki} \quad$ [doi:10.1250/ast.27.264]

\section{INTRODUCTION}

The feedforward active noise control system consists of three filters, namely, a noise control filter, a secondary path filter and a feedback control filter [1]. Only one of these filters, the noise control filter, has coefficients that are repeatedly updated. The coefficients of the other filters are fixed to the values estimated by feeding extra noise to a loudspeaker (secondary source), before starting active noise control. In practical systems, both the secondary and the feedback paths should be assumed to be continuously changing. This change inevitably degrades the performance of the system [2]. At worst, the system becomes uncontrollable. The coefficients of both the secondary path filter and the feedback control filters should be both refreshed at intervals.

The simplest way of refreshing the coefficients of the two filters is to feed the extra noise to the secondary source [3]. This feeding, however, makes the active noise control system a new noise source. The authors have already presented the "simultaneous equations method" for refreshing

\footnotetext{
*e-mail: er05i005@steng.u-hyogo.ac.jp

†e-mail: fujiken@eng.u-hyogo.ac.jp

†e-mail: muneyasu@ipeku.kansai-u.ac.jp
}

the coefficients of the secondary path filter without feeding the extra noise to the secondary source [4], and moreover, have applied the method to update the coefficients of the noise control filter without using the secondary path filter $[5,6]$.

The filtered-U algorithm [7] does not require the feedback control filter. This algorithm, however, is not applicable to systems with high feedback path gain. We hence propose a method of refreshing the coefficients of the feedback control filter without feeding the extra noise to the secondary source. One problem is that the only signal available for the refreshment is the output of the noise detection microphone. This output involves two unknowns related to the feedback path and the primary noise. To identify the feedback path, two independent equations are required.

We modify the simultaneous equations method [4-6] to provide the two independent equations from only the output of the noise detection microphone. The auxiliary filter [4-6] applied to the identification of the overall path from the noise detection microphone to the error sensor microphone is used for the linear prediction of the input signal of the noise control filter. The coefficients of the auxiliary filter then yield an equation consisting of the feedback path, noise control filter and the primary noise. 




Fig. 1 Typical configuration of feedforward active noise control system.

Here, the feedback path and the primary noise are unknown. The simultaneous equations method therefore gives different coefficients to the noise control filter; thereby the coefficients of the auxiliary filter can form two independent equations. The independent equations are solvable, and one of the solutions gives the impulse response of the feedback path.

Another problem is that the feedback component incident on the noise detection microphone is small in comparison with the primary noise impeding the identification of the feedback path. In this case, it takes a long time to identify the feedback path with high precision. To reduce the time, we paper present a method of estimating first the primary noise and then the feedback path.

\section{PRINCIPLE}

Figure 1 illustrates a typical configuration of feedforward active noise control system, where $\mathrm{Md}, \mathrm{Sp}, \mathrm{Me}$ and the $z$-transforms denote the following equipment, paths, filters and signals.

Md: Noise detection microphone

Me: Error sensor microphone

Sp: Loudspeaker

$N(z)$ : Primary noise

$P(z)$ : Primary path

$C(z)$ : Secondary path

$B(z)$ : Feedback path

$\hat{B}(z)$ : Feedback control filter

$H(z)$ : Noise control filter

$X(z)$ : Input signal of the noise control filter

$E(z)$ : Output of the error sensor microphone

In addition, the paths are assumed to have no zero.

As seen from the configuration, the feedback path from the loudspeaker to the noise detection microphone forms a closed loop. Figure 2 shows the block diagram of the closed loop. In this loop, the feedback control filter is inserted so as to cut off the loop. The gain of the loop decreases when the filter is modeled on the feedback path.

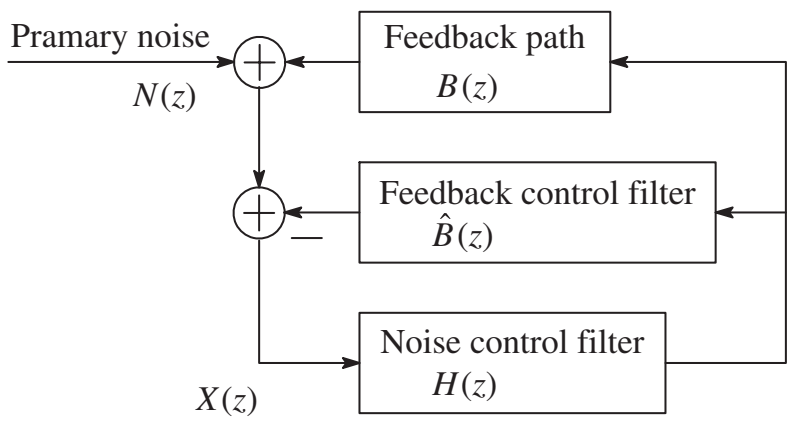

Fig. 2 Closed loop structured with the feedback path.

We present a method of estimating the coefficients of the feedback control filter that minimizes the gain.

The signal available for the estimation is $X(z)$, which can be expressed as

$$
X(z)=\frac{N(z)}{1-H(z)\{B(z)-\hat{B}(z)\}} .
$$

Here, $B(z)$ and $N(z)$ are unknown. In this case, it is impossible to estimate the coefficients of the feedback control filter minimizing the loop gain from only $X(z)$. To make the estimation possible, we first express the primary noise as follows:

$$
N(z)=\frac{W(z)}{1-Q(z)},
$$

where $W(z)$ is a linearly unpredicted process and

$$
Q(z)=\sum_{k=1}^{K} q(k) z^{-k}
$$

is a linearly predicted component.

In this expression, the input signal $X(z)$ can be expanded as

$$
\begin{aligned}
X(z) & =\frac{W(z)}{1-Q(z)} \cdot \frac{1}{1-H(z) \Delta B(z)} \\
& =\frac{W(z)}{1-[Q(z)+H(z) \Delta B(z)\{1-Q(z)\}]},
\end{aligned}
$$

where

$$
\Delta B(z)=B(z)-\hat{B}(z)
$$

In Eq. (4), our target $\Delta B(z)$ is involved in the denominator. To extract $\Delta B(z)$, we next apply the linear prediction analysis to $X(z)$. The application consequently provides the linear prediction filter whose transfer function is expressed as

$$
S(z)=Q(z)+H(z) \Delta B(z)\{1-Q(z)\} .
$$

However, Eq. (6) still involves two unknowns, namely, $\Delta B(z)$ and $Q(z)$. To ascertain $\Delta B(z)$, another independent equation is necessary. 
The simultaneous equations method [4-6] yields two independent equations by giving $\boldsymbol{H}_{1}$ and $\boldsymbol{H}_{2}$, which are different coefficient vectors, to the noise control filter. In this case, the linear prediction analysis provides two independent relations:

$$
S_{1}(z)=Q(z)+H_{1}(z) \Delta B(z)\{1-Q(z)\}
$$

and

$$
S_{2}(z)=Q(z)+H_{2}(z) \Delta B(z)\{1-Q(z)\},
$$

where $H_{1}(z)$ and $H_{2}(z)$ are $z$-transforms of the coefficient vectors $\boldsymbol{H}_{1}$ and $\boldsymbol{H}_{2}$, respectively. In addition, $\boldsymbol{H}_{1}$ and $\boldsymbol{H}_{2}$ naturally form different $H_{1}(z)$ and $H_{2}(z)$; thereby different $S_{1}(z)$ and $S_{2}(z)$ are obtained. In this case, Eqs. (7) and (8) are solvable; therefore eliminating $Q(z)$ from them gives the difference,

$$
\Delta B(z)=\frac{S_{1}(z)-S_{2}(z)}{H_{1}(z)\left\{1-S_{2}(z)\right\}-H_{2}(z)\left\{1-S_{1}(z)\right\}},
$$

which consists of the known $z$-transforms [8]. This is the principle of the method used in this paper.

\section{SEQUENTIAL ESTIMATION}

To apply this principle to practical systems, another modification is necessary. The primitive application severely delays the estimation of $\Delta B(z)$. This delay is caused by the error of the estimated $S(z)$. Actually, the feedback component incident on the noise detection microphone from the loudspeaker is extremely small in comparison with the primary noise, that is,

$$
Q(z) \gg H(z) \Delta B(z)\{1-Q(z)\}
$$

in Eq. (6). Moreover, the second term of Eq. (6) decreases whenever $\hat{B}(z)$ is estimated. Sooner or later, the second term will be buried in error. It thereby becomes difficult to estimate $\Delta B(z)$ with high precision. We hence propose a sequential technique of estimating first $Q(z)$ and then $\Delta B(z)$.

Figure 3 shows the configuration of the sequential estimation technique. In this technique, $Q(z)$ is estimated from Eqs. (7) and (8) as follows:

$$
\hat{Q}(z)=\frac{S_{2}(z) H_{1}(z)-S_{1}(z) H_{2}(z)}{H_{2}(z)-H_{1}(z)} .
$$

This estimated $\hat{Q}(z)$ is approximate to $Q(z)$ when $S_{1}$ and $S_{2}(z)$ are estimated with high precision.

As shown in Fig. 3, the technique first adds $X(z)$ to the

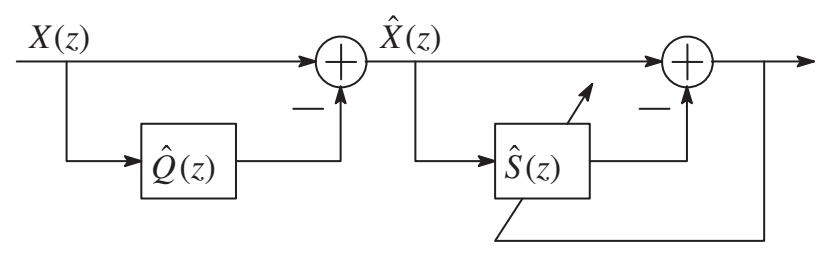

Fig. 3 Configuration of sequential estimation. former circuit whose transfer function is $1-\hat{Q}(z)$. The modified input signal, $\hat{X}(z)$, is thereby obtained as follows:

$$
\hat{X}(z)=X(z)\{1-\hat{Q}(z)\} .
$$

In addition, this $\hat{X}(z)$ can be rearranged by substituting Eq. (4) into Eq. (12) as follows:

$$
\hat{X}(z)=\frac{W(z)}{1-\hat{S}(z)},
$$

where

$$
\hat{S}(z)=\tilde{Q}(z)+H(z) \Delta B(z)\{1-\tilde{Q}(z)\}
$$

and

$$
\tilde{Q}(z)=\frac{Q(z)-\hat{Q}(z)}{1-\hat{Q}(z)} .
$$

Here, as mentioned above, since $\hat{Q}(z) \approx Q(z), \tilde{Q}(z) \approx 0$. This means that the second term is predominant over the first term in Eq. (14). Then, $\Delta B(z)$ can be extracted with high precision even if $\hat{S}(z)$ involves some error.

The sequential estimation technique applies the linear prediction analysis to $\hat{X}(z)$ after giving other different coefficient vectors $\boldsymbol{H}_{1}$ and $\boldsymbol{H}_{2}$ to the noise control filter. Thereby, two independent equations with $H_{1}(z)$ and $H_{2}(z)$ are obtained again as

$$
\hat{S}_{1}(z)=\tilde{Q}(z)+H_{1}(z) \Delta B(z)\{1-\tilde{Q}(z)\}
$$

and

$$
\hat{S}_{2}(z)=\tilde{Q}(z)+H_{2}(z) \Delta B(z)\{1-\tilde{Q}(z)\} .
$$

Here, as mentioned above, since $\tilde{Q}(z) \approx 0$, the second terms of Eqs. (16) and (17) are predominant over each first term. Consequently, eliminating $\tilde{Q}(z)$ from Eqs. (16) and (17) gives the difference,

$$
\Delta \hat{B}(z)=\frac{\hat{S}_{1}(z)-\hat{S}_{2}(z)}{H_{1}(z)\left\{1-\hat{S}_{2}(z)\right\}-H_{2}(z)\left\{1-\hat{S}_{1}(z)\right\}} .
$$

\section{FREQUENCY DOMAIN ESTIMATION}

In practical use, a process for transforming $\hat{P}(z)$ and $\Delta \hat{B}(z)$ into filter coefficient vectors is required. An identification technique for the transformation has been presented [8]. The processing cost of this technique, however, is extremely high. Here, we apply the frequency domain processing technique [6] to the transformation.

In the frequency domain, Eqs. (11) and (18) are expressed as

$$
\begin{gathered}
\hat{Q}(\omega)=\frac{S_{2}(\omega) H_{1}(\omega)-S_{1}(\omega) H_{2}(\omega)}{H_{2}(\omega)-H_{1}(\omega)} \\
\Delta \hat{B}(\omega)=\frac{\hat{S}_{1}(\omega)-\hat{S}_{2}(\omega)}{H_{1}(\omega)\left\{1-\hat{S}_{2}(\omega)\right\}-H_{2}(\omega)\left\{1-\hat{S}_{1}(\omega)\right\}},
\end{gathered}
$$

where $H_{1}(\omega)$ and $H_{2}(\omega)$ are the frequency responses of the 


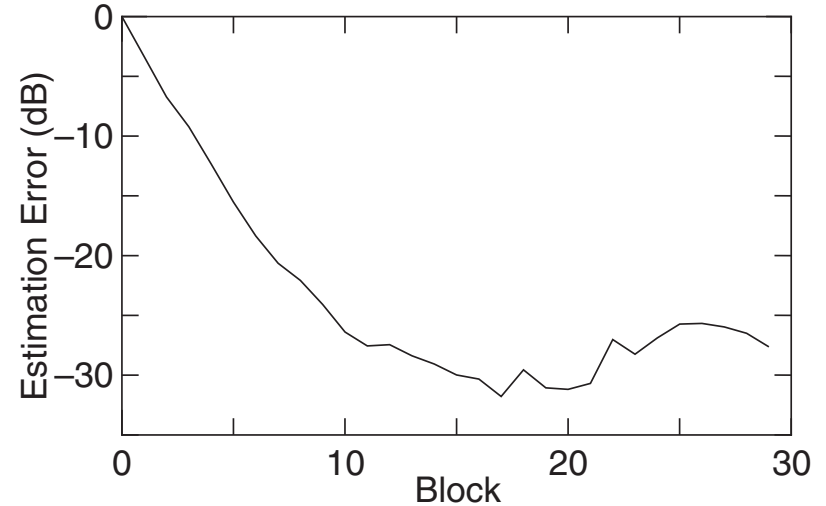

Fig. 4 Convergence property of the estimation error of the feedback path.

noise control filter, $S_{1}(\omega)$ and $S_{2}(\omega)$ are the frequency responses of the first linear prediction filter applied to $X(z)$, and $\hat{S}_{1}(\omega)$ and $\hat{S}_{2}(\omega)$ are the frequency responses of the second linear prediction filter applied to $\hat{X}(z)$. They all are obtained as the Fourier transforms of the filter coefficient vectors. Moreover, the transformations of $\hat{Q}(z)$ and $\Delta \hat{B}(z)$ to filter coefficient vectors can be calculated as the inverse Fourier transforms of $\hat{Q}(\omega)$ and $\Delta \hat{B}(\omega)$.

Figure 4 gives a simulation result calculated under the following conditions.

(1) The primary noise is generated as the output of a filter with the transfer function

$$
F(z)=\frac{1}{1-2 \gamma \cos \theta z^{-1}+\gamma^{2} z^{-2}},
$$

where $\gamma=0.99$, and $\theta=\pi / 4$ corresponding to the resonance frequency of $1 \mathrm{kHz}$ when the sampling frequency is $8 \mathrm{kHz}$. This output also approximates jet fan noise when the input signal of the filter is white noise.

(2) The number of impulse response samples of the feedback path is 32 , and also, regular random numbers are given as the impulse response samples.

(3) The element numbers of the coefficient vectors of the noise control filter and the linear prediction filter are 32 and 128 , respectively.

(4) The adaptive algorithm used for the estimations of $S(z)$ and $\hat{S}(z)$ is the NLMS algorithm whose step sizes are 0.01 and 0.001 , respectively.

(5) The transformations of $\hat{Q}(z)$ and $\Delta \hat{B}(z)$ to the filter coefficient vectors are performed after iterating the estimations of $S_{1}(z), S_{2}(z), \hat{S}(z)$ and $\hat{S}(z) 50,000$ times each. Accordingly, one block in the horizontal axis corresponds to $50,000 \times 4=200,000$ iterations.

This result shows that the proposed method can identify the feedback path with an estimation error less than $-25 \mathrm{~dB}$ after ten blocks corresponding to 2,000,000 iterations. This number of iterations necessary for the estimation

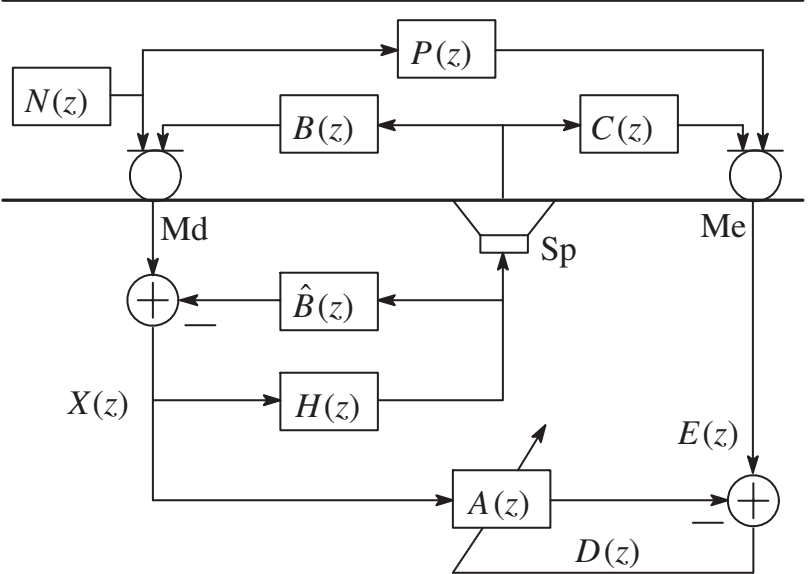

Fig. 5 Active noise control system using simultaneous equations method.

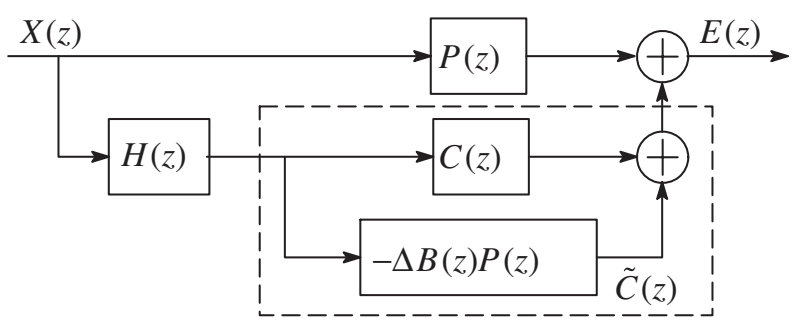

Fig. 6 Overall path identified by auxiliary filter.

is extremely low in comparison with the method presented in [8].

\section{CONDITION FOR OBTAINING THE NOISE REDUCTION EFFECT}

We next examine the influence of the identification error involved in the coefficients of the feedback control filter on the noise reduction effect provided by the active noise control system using the simultaneous equations method. Here, the configuration of the active noise control system is shown in Fig. 5. As seen from this configuration, the simultaneous equations method is characterized by the auxiliary filter $A(z)$ identifying the overall path from the input of the noise control filter to the output of the error sensor microphone.

Figure 6 also illustrates the configuration of the overall path. After the overall path identification, the auxiliary filter gives the relation

$$
A(z)=P(z)+\tilde{C}(z) H(z),
$$

where

$$
\tilde{C}(z)=C(z)-\Delta B(z) P(z) .
$$

Naturally, $A(z)$ shown in Eq. (22) involves two unknowns, $P(z)$ and $\tilde{C}(z)$. The simultaneous equations method hence gives two different coefficient vectors to the noise control 
filter and individually identifies the overall path as

$$
A_{1}(z)=P(z)+\tilde{C}(z) H_{1}(z)
$$

and

$$
A_{2}(z)=P(z)+\tilde{C}(z) H_{2}(z) .
$$

Here, since $H_{1}(z) \neq H_{2}(z)$, the two solutions

$$
\tilde{C}(z)=\frac{A_{1}(z)-A_{2}(z)}{H_{1}(z)-H_{2}(z)}
$$

and

$$
P(z)=\frac{A_{1}(z) H_{2}(z)-A_{2}(z) H_{1}(z)}{H_{2}(z)-H_{1}(z)}
$$

can be obtained. In addition, the optimum noise control filter, which reduces the error sensor output to zero, satisfies the relation

$$
P(z)+\tilde{C}(z) H(z)=0 .
$$

Finally, substituting (26) and (27) into (28) yields the transfer function of the optimum noise control filter as follows:

$$
H_{\mathrm{opt}}(z)=\frac{A_{1}(z) H_{2}(z)-A_{2}(z) H_{1}(z)}{A_{1}(z)-A_{2}(z)} .
$$

Figure 7 shows the relationship between the noise reduction effect and the identification error of the feedback path, where this result is calculated under the following conditions.

(1) The number of impulse response samples of the primary path is 64 .

(2) The element number of the coefficient vector of the auxiliary filter is 64 .

(3) The frequency domain adaptive algorithm,

$$
A_{n+1}(k)=A_{n}(k)+\mu \frac{\sum_{j=n J+1}^{(n+1) J} D_{j}(k) X_{j}^{*}(k)}{\sum_{j=n J+1}^{(n+1) J} X_{j}(k) X_{j}^{*}(k)},
$$

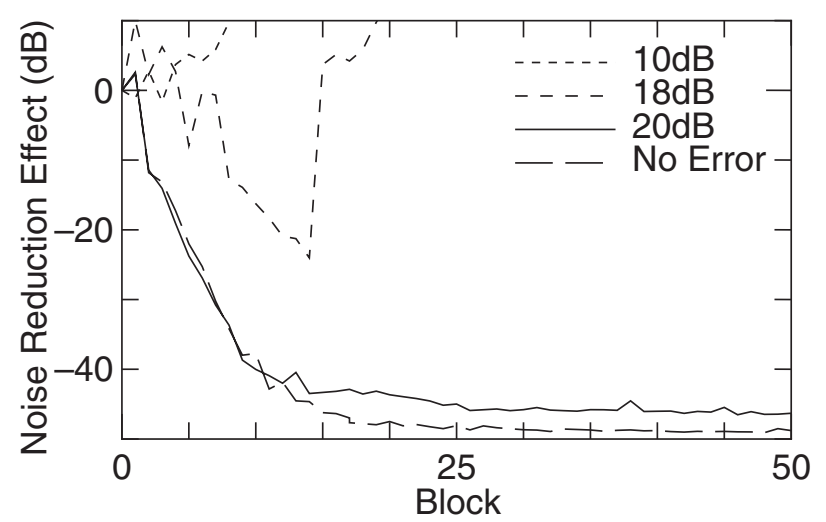

Fig. 7 Relationship between noise reduction effect and identification of error of feedback path. is used for the identification of the overall path, where $n$ is the block number, $k$ is the element number of the Fourier transform, $j$ is the Fourier transform duration number, $J=5, \mu=0.75$, and $D_{j}(k)$ and $X_{j}(k)$ are $k$ th elements of the Fourier transforms of the identification error and the input signal of the noise control filter, respectively.

(4) The coefficient vector of the noise control filter is estimated whenever $A_{n}(k)$ is updated five times. Accordingly, one block in the horizontal axis corresponds to 2,560 sampling times.

In the result shown in Fig. 7, the output power of the error sensor microphone increases when the identification error is $-18 \mathrm{~dB}$ or greater. The increase indicates the occurrence of howling caused by the feedback path. This means that the simultaneous equations method can provide a sufficient noise reduction effect unless howling occurs.

\section{FREQUENCY DOMAIN PROCESSING}

It is well known that frequency domain adaptive algorithms provide higher estimation speeds than time domain adaptive algorithms. We improve the estimation speed by applying the following frequency domain adaptive algorithm,

$$
S_{n+1}(k)=S_{n}(k)+\mu_{n}(k) \sum_{j=n J+1}^{(n+1) J} G_{j}(k) X_{j}^{*}(k),
$$

to the linear prediction analysis, where $G_{j}(k)$ is the linear prediction error. In the application of the frequency domain adaptive algorithm to the linear prediction analysis, it should be noted that the impulse response of the closed loop continues infinitely. This means that a very long Fourier transform duration is necessary for the linear prediction. This long duration naturally delays the estimation of $S_{n}(k)$. To decrease the delay, we use the following step size control,

$$
\mu_{n}(k)=\frac{\sum_{j=n J+1}^{(n+1) J} G_{j}(k) G_{j}^{*}(k)}{\sum_{j=n J+1}^{(n+1) J} \sum_{k=0}^{K} G_{j}(k) G_{j}^{*}(k)} \times \alpha,
$$

where $\alpha$ is an integer, and $K$ is the total element number of the Fourier transform. By applying this adaptive algorithm to the linear prediction, the estimation speed is expected to increase.

Figure 8 is a simulation result calculated to examine whether the estimation speed can be improved by using the frequency domain adaptive algorithm, where $J=2, \alpha=$ 0.002 for the analysis of $S(z), \alpha=0.0002$ for the prediction of $\hat{S}(z)$, the number of iterations of updating $S_{n}(k)$ is 781 (accordingly, one block in the horizontal axis corresponds 


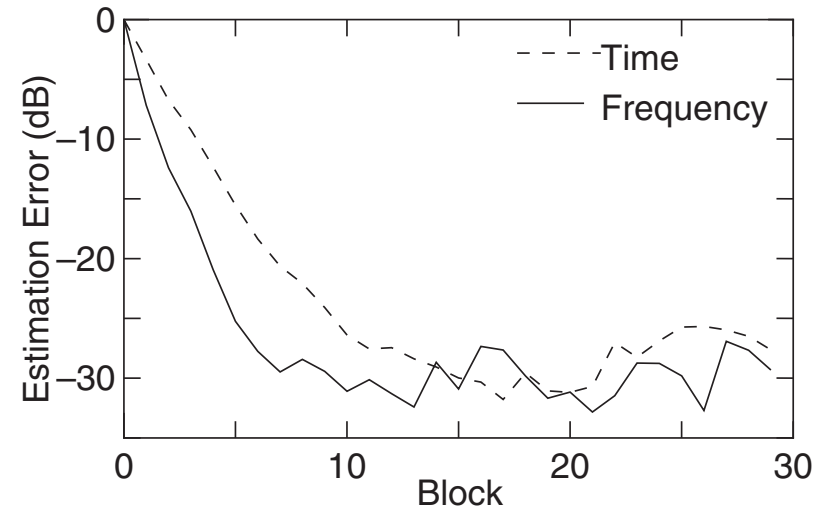

Fig. 8 Convergence speeds obtained by the time and the frequency domain adaptive algorithms.

to 199,936 ), and the other conditions are equal to those in Fig. 4. This result shows that the application of the frequency domain adaptive algorithm to the identification of the feedback path is valid.

\section{CONCLUSIONS}

We have proposed a method for identifying the feedback path without feeding extra noise to the secondary source and presented a sequential estimation technique capable of estimating the feedback component buried in the primary noise. The simulation results have also shown that the proposed method and technique can be applied to estimate the coefficients of the feedback control filter with an error less than $-20 \mathrm{~dB}$.

Our subsequent studies will focus on the verification of the presented results with an experimental system.

\section{REFERENCES}

[1] S. J. Elliott and P. A. Nelson, "Active noise control," IEEE Signal Process. Mag., 10, 12-35 (1993).

[2] S. D. Synder and C. H. Hansen, "The effect of transfer function estimation errors on the filtered-x LMS algorithm," IEEE Trans. Signal Process., SP-42, 950-953 (1994).

[3] L. J. Erikson and M. C. Allie, "Use of random noise for on-line transducer modeling in an adaptive active attenuation system," J. Acoust. Soc. Am., 85, 797-802 (1989).

[4] K. Fujii and J. Ohga, "Method to update the coefficients of the secondary path filter under active noise control," Signal Process., 81, 381-387 (2001).

[5] K. Fujii, M. Muneyasu and J. Ohga, "Simultaneous equations method not requiring the secondary path filter," Proc. ACTIVE 99, pp. 941-948 (1999).

[6] K. Fujii, H. Hashimoto and M. Muneyasu, "Application of a frequency domain processing technique to the simultaneous equation method," IEICE Trans. Fundam., E86-A, 2020-2027 (2003).

[7] L. J. Eriksson, "Development of the filtered-U algorithm for active noise control," J. Acoust. Soc. Am., 89, 257-265 (1991).

[8] K. Fujii, M. Muneyasu and J. Ohga, "A method to update the coefficients of feedback control filter under active noise control," IEICE, J82-A, 843-850 (1999). 